\title{
The colours of bacteria and fungi
}

\author{
Ersilia Vita Fiscarelli \\ 'Bambino Gesù' Paediatric Hospital, Rome, Italy
}

Talking about colours, a bacteriology laboratory can be compared to a painter's studio: culture media are like canvasses, bacteria and fungi like paints and sterile loops look like extraordinary paintbrushes. Even Sir Alexander Fleming, the father of penicillin, before making his discovery, took pleasure in painting. As a member of the Chelsea Arts Club, besides the classical watercolours, he also painted dancers, horses, soldiers, breastfeeding mothers and other subjects, using live microorganisms (bacteria) instead of more conventional oil paints. The persistence of these works, obtained by growing bacteria with different natural pigments in Petri dishes, depended on the time necessary for a species of bacteria, livelier than others, to overcome the boundary between "a hat and a face", thus confounding the line between art and science (Figure 1).

Microbes are invisible to the naked eye. They become perceivable as develop into microbial "colonies" made up of millions and millions of individuals, that can take on various colours due to the release into the agar of diffusible pigments or to the production of specific metabolites during the colonies' growth (Figure 2).

A colour, or the absence of colour in a biological material culture, is of fundamental importance in guiding microbiologists in the vast microbial universe, leading them along the path of diagnosis. Even the different shades of colour are important: strawyellow is different from ochre-yellow, salmon pink from orange, as well as strawberry red from purple red.

Many, really many are the colours of microbes.

Staphylococcus aureus is a very common microorganism that may be responsible for skin and other infections; its name already

Correspondence: Ersilia Vita Fiscarelli, Ospedale Pediatrico Bambino Gesù, Piazza di Sant'Onofrio 4, 00165 Roma (RM), Italia.

E-mail: evita.fiscarelli@opbg.net

Key words: Colours, Laboratory, Bacteria, Fungi.

Conflict of interest: the author declares no potential conflict of interest.

Funding: none.

Received for publication: 21 October 2019.

Accepted for publication: 21 October 2019.

${ }^{\circ}$ Copyright: the Author(s), 2019

Licensee PAGEPress, Italy

Microbiologia Medica 2019; 34:8631

doi:10.4081/mm.2019.8631

This article is distributed under the terms of the Creative Commons Attribution Noncommercial License (by-nc 4.0) which permits any noncommercial use, distribution, and reproduction in any medium, provided the original author(s) and source are credited. stresses the "golden-yellow" colour that is conferred to the colonies by zeaxanthin, a yellow-orange pigment belonging to the carotenoids family.

Even pus from wounds infected by this microorganism is yel-
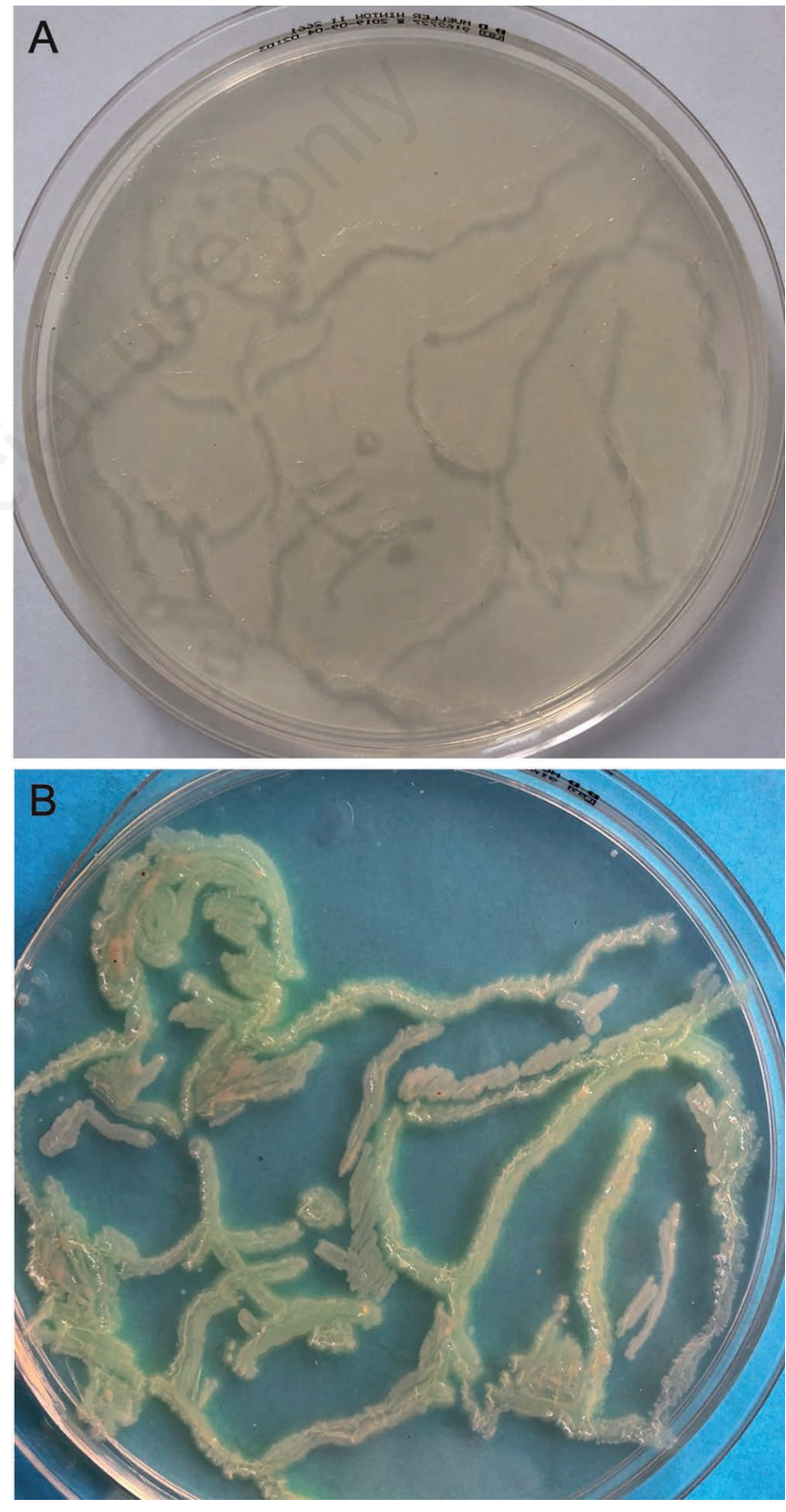

Figure 1. Creation of Adam: particular after 4 and 24 hours of incubation (respectively $A$ and $B)$. 


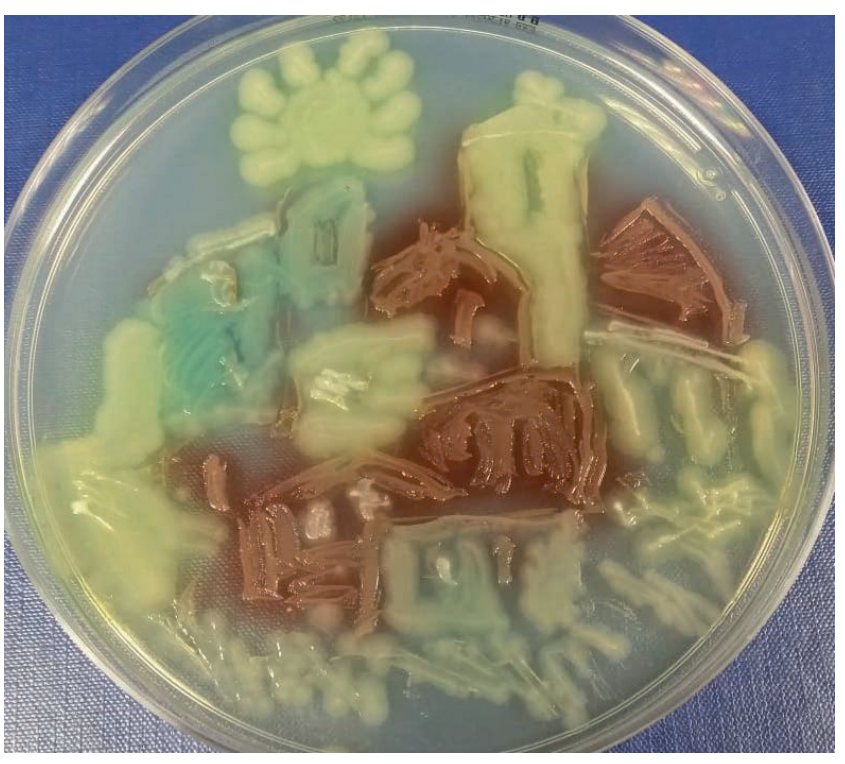

Figure 2. Landscape (various colours due to the release into the agar of diffusible pigments).

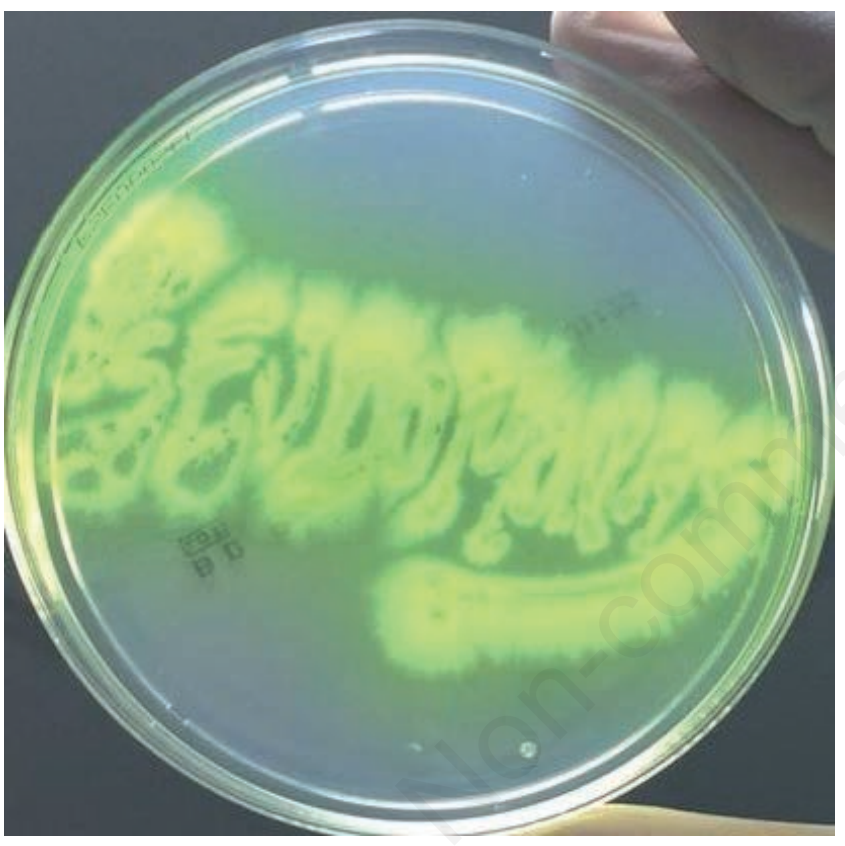

Figure 3. Pseudomonas aeruginosa on Pseudosel agar.

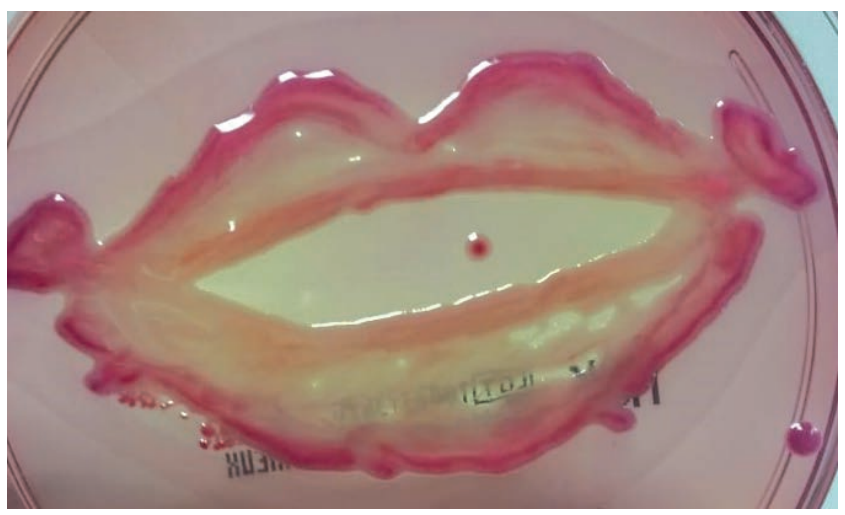

Figure 4. Lips (pale-pink to wine-red of Enterobacteriaceae). low. Galeno (131-201 B.C.) one of the fathers of ancient medicine and a strong supporter of the "humor" theory, defined it "bonum et laudabile", considering it "the materia peccans" that must be eliminated, in contrast with the grey-black colour of the gangrenous eschars caused by anaerobic bacteria which, in the pre-antibiotic era, implied the certainty of an inauspicious prognosis.

Blue-green, sometimes with metal-like reflections, is the colour of the colonies of Pseudomonas aeruginosa, a ubiquitarian in nature microbe that can cause not only skin and systemic infections in burn patients, but in other sites, too. This microorganism is also called "bacillus pyocyaneus" due to the peculiar bluish colour of the pus arising from the infected wounds, caused by the presence of the pigment pyocyanin. Pseudomonas aeruginosa also produces another pigment, pyoverdin, which determines the greenish hue. Both pigments are important virulence factors, as they increase the pathogen's capacity to cause damage in the host (Figure 3).

Almost infinite are the different shades of colours found in the Enterobacteriaceae colonies, varying from pale-pink to wine-red (Figure 4).

Enterobacteriaceae family includes several microorganisms belonging to different genera and species, some of which constitute the gut microbiota. For such bacteria, detecting in the culture pinkish-red colonies suggests a positive diagnostic significance, as it allows to exclude the presence of enteropathogenic microbes, such as Salmonella, whose colonies are transparent.

However, the diagnostic context in which colour expresses its greatest suggestiveness is mycology. Still today, though being in a genomic era, the systematic classification of the fungi with clinical relevance is mainly based on the observation of their phenotypical characteristics: the different fungal species are classified according to the macroscopic and microscopic features. For the assessment of the macroscopic aspects of the filamentous fungi for diagnostic purposes, the colony diameter, morphology, margins, surface texture (velvety, dusty, flacky, etc..) are extremely relevant elements. However, it is the colour of the aerial part of the colony, condi-

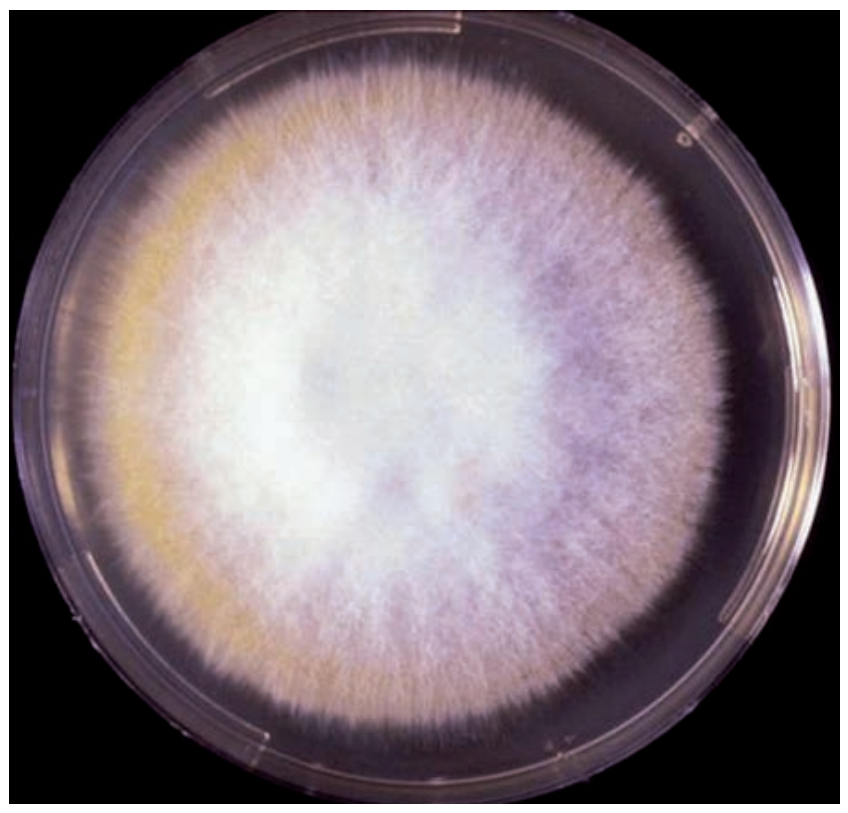

Figure 5. Microsporum canis (a peripheric margin of brilliant lemon-yellow due to the presence of a pigment). 

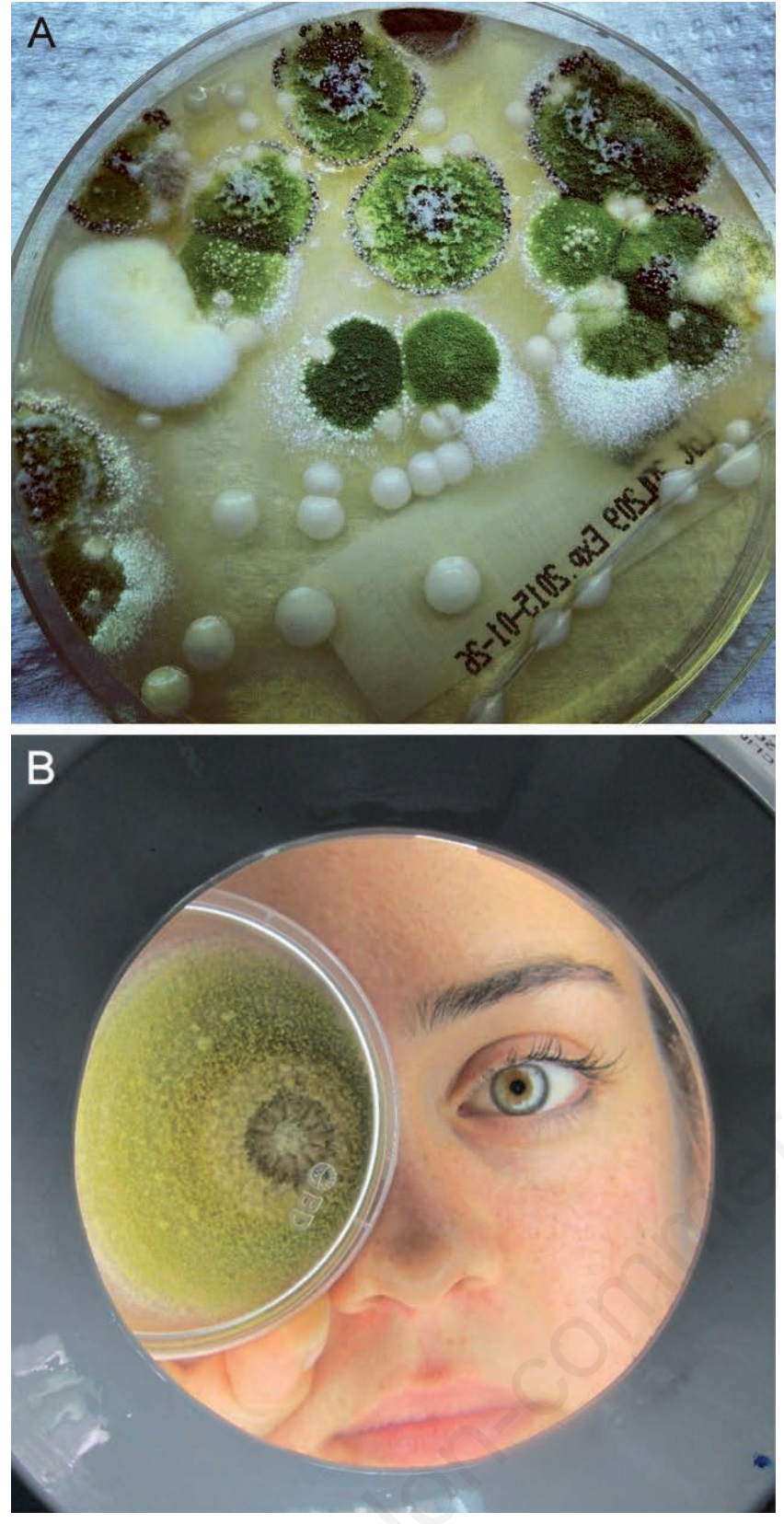

Figure 6. The green, blue-green of Aspergillus fumigatus (A) and the yellow-green of Aspergillus flavus (B). tioned by the vegetative mycelium, that constitutes one of the most important features, universally recognised as a characterizing element of the species.

In the Aspergillus genus, the green, blue-green, grey-turquois of the mature colonies of Aspergillus fumigatus are different from the yellow-green of Aspergillus flavus, from the creamy-white of Aspergillus candidus, from the black of the colonies of Aspergillus niger, as well as from many others (Figure 5).

Even for recognizing Dermatophytes fungi able to metabolize keratin and, therefore of great interest to dermatologists, the colour and shades of the colonies are extremely important features.

Microsporum canis is a zoophile fungus responsible for the infections of dogs, cats and other domestic animals. In humans, especially children, it can give rise to clinical pictures that are characteristic of tinea capitis, tinea corporis and, less frequently, tinea unguium. The colony colour varying from creamy white to suedelike colour with a peripheric margin of brilliant lemon-yellow due to the presence of a pigment which diffuses in agar, is so characteristic as to be pathognomonic (Figure 6).

Trichophyton rubrum is one of the isolated dermatophytes that is most commonly found in human lesions, where it parasitizes nails, skin and, less frequently, hair and beard. In this case, it is the characteristic port-red colouring with its diffuse melanoid pigmentation on the flip side of the colony thus suggesting the diagnosis, like the red-purple colonies with a velvety texture, to be suggestive of Trichophyton violaceum.

The world of microbes is a kaleidoscope: let's take the opportunity to appreciate also the beauty of organisms that are otherwise feared (sometimes rightly) or even ignored. 